Article

\title{
Investigation of Antimicrobial Peptide Genes Associated with Fungus and Insect Resistance in Maize
}

\author{
Joseph Noonan ${ }^{1}$, William Paul Williams ${ }^{2}$ and Xueyan Shan ${ }^{1, *}$ \\ 1 Department of Biochemistry, Molecular Biology, Entomology and Plant Pathology, Mississippi State \\ University, Mississippi State, MS 39762, USA; jn377@msstate.edu \\ 2 USDA-ARS, Corn Host Plant Resistance Research Unit, Mississippi State, MS 39762, USA; \\ paul.williams@ars.usda.gov \\ * Correspondence: xshan@bch.msstate.edu; Tel.: +1-662-325-5217
}

Received: 16 August 2017; Accepted: 6 September 2017; Published: 15 September 2017

\begin{abstract}
Antimicrobial peptides (AMPs) are small defense proteins present in various organisms. Major groups of AMPs include beta-barrelin, hevein, knottin, lipid transfer protein (LTP), thionin, defensin, snakin, and cyclotide. Most plant AMPs involve host plant resistance to pathogens such as fungi, viruses, and bacteria, whereas a few plant AMPs from the cyclotide family carry insecticidal functions. In this research, a genome-wide investigation on antimicrobial peptide genes in maize genome was conducted. AMPs previously identified from various plant species were used as query sequences for maize genome data mining. Thirty-nine new maize AMPs were identified in addition to seven known maize AMPs. Protein sequence analysis revealed 10 distinguishable maize AMP groups. Analysis of mRNA expression of maize AMP genes by quantitative real-time polymerase chain reaction (qRT-PCR) revealed different expression patterns in a panel of 10 maize inbred lines. Five maize AMP genes were found significantly associated with insect or fungus resistance. Identification of maize antimicrobial peptide genes will facilitate the breeding of host plant resistance and improve maize production.
\end{abstract}

Keywords: plant antimicrobial peptides; defensin; hevein; snakin; cyclotide; maize; host plant resistance

\section{Introduction}

Plants are constantly under attack by insect pests as well as bacterial, viral, and fungal pathogens. Various plant defense mechanisms exist to build up a complex and efficient innate plant immune system. The innate plant defense system includes the constitutive defense, which is the primary form of surveillance to provide first-line protection from pathogens, and the induced defense, which is triggered through signal transduction and defense gene expression upon insect feeding or pathogenic infection. The induced plant defense system responds to the pathogenic infection and promotes the production of defense-related secondary metabolites and pathogenesis-related proteins (PR proteins). Plant AMPs are classified as PR proteins with major types including beta-barrelins, heveins, knottins, lipid transfer proteins (LTPs), thionins, defensins, snakins, and cyclotides [1-4]. The types of plant AMPs present in a plant vary from species to species. Reports of plant AMPs have represented a few plant families, such as Amaranthaceae, Andropogoneae, Brassicaceae, Oryzeae, Solanaceae, Triticeae, and Violaceae [5-8].

Plant AMPs are peptides or low molecular weight proteins either constitutive or induced to deliver attacks to plant pathogens. Most plant AMPs identified to date demonstrated resistance to fungi, viruses, and bacteria, with only a few AMPs from the cyclotide family showing growth inhibition 
effects to insect larvae [2,9-12]. Plant AMPs eliminate pathogens by penetrating and interfering with the structural components of cell membranes. Many plant AMPs function by embedding themselves in the microbial cell membrane and forming pore-like membrane openings that cause leakage of essential ions and nutrients which ultimately leads to cell death $[1,12,13]$. Plant AMPs possess common characteristics and conserved structures, featuring a characteristic cysteine-rich motif. The conserved cysteine residues are the sites to form multiple disulfide bonds. They are generally peptides or small proteins having less than 90 amino acids in length. Many of them are basic proteins, carrying net positive charges in physiological neutral cellular environments. Despite the highly conserved protein structures, the genes coding for plant AMPs are highly polymorphic. In general, they are genes encoded by two or three exons, with each exon encoding for a different domain. Only the cysteine-rich domains will be present in the mature protein $[1-3,14,15]$. The unique characteristics and conserved structures of AMPs allow them to be easily recognized and grouped into different AMP families. While most are linear, cyclic AMPs exist including bacteriocins in bacteria, theta-defensins in animals, and cyclotides in plants. Many AMPs are rich in cysteine residues which function to stabilize the tertiary structure of these peptides through the cross-bracing of multiple disulfide bonds. The resulting compact structures exhibit high thermal, chemical, and enzymatic stability. Many linear antimicrobial peptides are near cyclic due to their multiple disulfide bonds $[1,11]$. Their subsequent compact structures similarly result in high thermal, chemical, and enzymatic stability. The sequences of plant AMPs identified to date are available from the databases UniProt and PhytAMP, allowing the search of AMP sequence information and biological data. These databases also provide bioinformatics analysis tools to enhance the understanding of plant AMPs [5,16].

AMPs are classified based on their cysteine motifs, sequence similarity, and conserved secondary and tertiary structures. Plant defensins are an abundant family of plant AMPs. Rich in cysteine residues, plant defensins are cationic proteins between $45-54$ amino acids. Triple-stranded antiparallel $\beta$-sheets and an $\alpha$-helix make up the structural conformation essential to the antimicrobial activity of plant defensins. These complex structures are stabilized by 4 to 5 disulfide bridges. Plant defensins have diverse functions including $\alpha$-amylase and trypsin inhibitory activity in addition to the typical AMP functions. Furthermore, the cationic nature of plant defensins facilitates interaction with sphingolipids and phospholipids of fungal membranes, ultimately disrupting homeostasis [1,17-19]. Through an unelucidated mechanisms, retarded cell growth or cell death occurs in fungus cells because of plant defensin interactions. Some proposed mechanisms suggest plant defensins initiate an autophagy-like response, or they induce reactive oxygen species within pathogenic fungi [18]. Heveins and hevein-like peptides are glycine-rich AMPs. They are basic peptides roughly 29-45 amino acids long with 3 to 5 disulfide bonds. Heveins bind to chitin and function to inhibit the growth of chitin-containing fungi thereby conferring defense against a plethora of fungal pathogens [1].

Knottin-type peptides make up a large group including the cyclotide family. Cyclotides are antimicrobial peptides only identified in plants. They are the only class of plant AMPs that demonstrate insecticidal activities $[1,5,10,12,20]$. Cyclotides are a family of plant AMPs with unique head to tail cyclized peptide structure. With the help of computational analysis and database screening, cyclotides have been identified mainly from Rubiaceae, Violaceae and Poaceae families [1,21]. They are small disulfide-rich proteins that have the unusual feature of a cyclic backbone and a cysteine knot. This protein consists of three disulfide bonds with connecting backbone segments which forms a ring in the structure that is penetrated by its third disulfide bond. They are among the smallest AMPs, 29-37 amino acids long, and the most diverse in function $[1,10,11]$. They can have hormone-like functions or activities related to enzyme inhibition, as well as cytotoxic, antimicrobial, insecticidal, and anti-HIV activities [1,10-12,22]. Despite the highly conserved motif, knottin-type peptides have hypervariable sequences. Due to the high sequence tolerance and diverse biological function, the knottin scaffold has even been used as a template for drug design. Cyclotides are particularly stabile due to their cyclic cysteine knot motif and cyclization. They are resistant to gastrointestinal proteases like trypsin, chymotrypsin, pepsin, and elastase. In addition, the cyclization results in limited 
unfolding thereby increasing resistance to elevated temperatures. The resulting topology formed by the cyclized backbone and the cysteine knot make cyclotides a highly unusual and interesting class of protein $[1,3,9,12,20,21]$.

Lipid transfer proteins (LTPs) are cationic proteins roughly 20-25 amino acids long with 4 disulfide bonds. They are particularly known for their lipid transfer activity, binding to a wide variety of lipids. These proteins promote membrane permeability in pathogens rather than host cells. Rich in lysine and arginine, $\alpha$-hairpinins are plant defense peptides roughly 33 amino acids long. Well documented for antibacterial and antifungal activities, $\alpha$-hairpinins inhibit spore germination and hyphal elongation of several plant pathogenic fungi in vivo. These are most known for their helix-loop-helix motif. Snakins have six disulfide bonds and are no longer than 66 amino acids. They are a component of the constitutive and inducible plant defense barriers and are known for their efficacious antifungal and antibacterial activities at low concentrations [23].

Plant AMPs are naturally occurring antimicrobial proteins expressed in various plant species. A thorough understanding of the occurrence and distribution of plant AMPs in crops is of great importance to increase crop yield and reduce plant diseases. The objectives of this research are to conduct a genome-wide investigation on maize AMP genes and examine their expression levels using maize inbred lines associated with various fungus and insect resistance. Especially, objectives of this research are to characterize and understand maize AMPs, their potential antimicrobial functions, and the number and distribution of AMPs in maize genome. Identification of maize antimicrobial proteins against agriculturally important insects and fungi will provide great insights and powerful methods for maize protection.

\section{Results}

\subsection{Identification of Maize AMPs}

Plant AMPs from PhytAMP Database [5] were used to BLAST the maize B73 genome database MaizeGDB [24,25]. There was a total of 271 plant AMPs from various plant families including Amaranthaceae, Andropogoneae, Brassicaceae, Oryzeae, Triticeae, and Violaceae curated in the PhytAMP database [5]. Of the 271 sequences, 189 plant AMP sequences were used to BLAST the whole maize B73 genome. Thirteen out of all the plant AMPs from the PhytAMP Database from various species yielded significant hits from the BLAST of whole maize B73 genome sequence (Table 1). These plant AMP sequences included 2 heveins from Beta vulgaris and Eutrema wasabi, respectively, 3 snakins each from Fagus sylvatica, Fragaria ananassa, and Zea mays, respectively, 2 LTPs from Zea mays, 2 defensins from Arabidopsis thaliana, a defensin from Zea mays, a beta-barrelin from Macadamia integrifolia, and 2 cyclotides from Chassakia parviflora and Viola hederacea, respectively (Table 1). A total of 46 maize protein sequences with homology to known plant AMPs were identified (Table 1). This included 39 new maize AMPs and 7 known maize AMPs. In some cases, 2 to 3 maize AMP sequences were found to derive from the same gene which produces variable lengths of protein sequences, such as GRMZM2G005633P1 and GRMZM2G005633P2 (Table 1). All of these variable versions appeared to be full length and were located to the same chromosome loci, with some of these loci close to transposable elements. All these sequences were considered as full-length maize AMPs and were included in further analysis. This resulted in the identification of 8 maize heveins, 12 maize snakins, 7 maize LTPs, 15 maize defensins, 1 maize beta-barrelin, and 3 maize cyclotides. Therefore, the B73 genome contains six potential AMP families. The names of the identified maize AMPs followed the Gramene ID system from MaizeGDB (Table 1). Maize AMPs were found on each of the 10 maize chromosomes (Table 1). The protein sequences of maize AMPs ranged from 35 aa (defensin GRMZM2G153488) to 379 aa (hevein GRMZM2G145528). The protein sequences of all the identified maize AMPs were summarized in Table S1. 
Table 1. Maize antimicrobial peptides (AMP) genes identified by basic alignment search tool (BLAST) maize B73 genome with known plant AMP sequences from various species.

\begin{tabular}{|c|c|c|c|}
\hline Maize Gene ID & Maize Chromosome & BLAST From Seq & Origin \\
\hline \multicolumn{4}{|c|}{ Hevein } \\
\hline GRMZM2G005633P1 & Ch10 & ctb1-chitinase B1 & Beta vulgaris \\
\hline GRMZM2G005633P2 & Ch10 & ctb1-chitinase B1 & Beta vulgaris \\
\hline GRMZM2G373106 & Ch8 & ctb1-chitinase B1 & Beta vulgaris \\
\hline GRMZM2G117942 & Ch4 & Q8H950 & Eutrema wasabi \\
\hline GRMZM2G145518 & Ch6 & Q8H950 & Eutrema wasabi \\
\hline GRMZM2G051921 & $\mathrm{Ch} 2$ & Q8H950 & Eutrema wasabi \\
\hline GRMZM2G051943 & $\mathrm{Ch} 2$ & cta1-chitinase & Beta vulgaris \\
\hline GRMZM2G117971 & Ch4 & Q8H950 & Eutrema wasabi \\
\hline \multicolumn{4}{|c|}{ Snakin } \\
\hline GRMZM2G105364 & Ch9 & Q0VYL5 & Fagus sylvatica \\
\hline GRMZM2G068202 & Ch2 & Q19VG5 & Zea mays \\
\hline GRMZM2G117940 & Ch1 & O49134 & Fragaria ananassa \\
\hline GRMZM2G172596 & Ch10 & Q0VYL5 & Fagus sylvatica \\
\hline GRMZM2G062527P1 & Ch6 & Q19VG5 & Zea mays \\
\hline GRMZM2G062527P2 & Ch6 & Q19VG5 & Zea mays \\
\hline GRMZM2G062527P3 & Ch6 & Q19VG5 & Zea mays \\
\hline GRMZM2G062527P4 & Ch6 & Q19VG5 & Zea mays \\
\hline GRMZM2G062527P5 & Ch6 & Q19VG5 & Zea mays \\
\hline GRMZM2G077034 & Ch5 & O49134 & Fragaria ananassa \\
\hline GRMZM2G164090 & Ch6 & Q19VG5 & Zea mays \\
\hline GRMZM2G107003 & Ch2 & Q19VG5 & Zea mays \\
\hline \multicolumn{4}{|c|}{ LTP } \\
\hline GRMZM2G010868P1 & Ch3 & Q2XX14 & Zea mays \\
\hline GRMZM2G010868P2 & Ch3 & $\mathrm{Q} 2 \times X 14$ & Zea mays \\
\hline GRMZM2G101958 & Ch10 & Q2XX14 & Zea mays \\
\hline GRMZM5G898755P1 & Ch10 & $\mathrm{Q} 2 \mathrm{XX14}$ & Zea mays \\
\hline GRMZM5G898755P2 & Ch10 & Q2XX14 & Zea mays \\
\hline GRMZM2G107839P1 & Ch3 & $\mathrm{Q} 2 \times \times 25$ & Zea mays \\
\hline GRMZM2G107839P2 & Ch3 & Q2XX25 & Zea mays \\
\hline \multicolumn{4}{|c|}{ Defensin } \\
\hline GRMZM2G368890 & Ch10 & P81008 & Zea mays \\
\hline GRMZM2G392863P1 & Ch1 & Q9ZUL7 & Arabidopsis thaliana \\
\hline GRMZM2G392863P2 & Ch1 & Q9ZUL7 & Arabidopsis thaliana \\
\hline GRMZM2G146809 & Ch10 & Q9ZUL7 & Arabidopsis thaliana \\
\hline GRMZM5G896902 & $\mathrm{Ch} 2$ & Q9ZUL7 & Arabidopsis thaliana \\
\hline GRMZM2G153488P1 & Ch5 & P82781 & Arabidopsis thaliana \\
\hline GRMZM2G153488P2 & Ch5 & P82781 & Arabidopsis thaliana \\
\hline GRMZM2G153488P3 & Ch5 & P82781 & Arabidopsis thaliana \\
\hline GRMZM2G064698P1 & Ch2 & Q9ZUL7 & Arabidopsis thaliana \\
\hline GRMZM2G064698P2 & Ch2 & Q9ZUL7 & Arabidopsis thaliana \\
\hline GRMZM2G153368P1 & Ch5 & P82781 & Arabidopsis thaliana \\
\hline GRMZM2G153368P2 & Ch5 & P82781 & Arabidopsis thaliana \\
\hline GRMZM2G368861P1 & Ch10 & P81008 & Zea mays \\
\hline GRMZM2G368861P2 & Ch10 & P81008 & Zea mays \\
\hline GRMZM2G046532 & Ch7 & P81008 & Zea mays \\
\hline \multicolumn{4}{|c|}{ Beta-Barrelin } \\
\hline GRMZM2G430500 & Ch2 & P80915 & Macadamia integrifolia \\
\hline \multicolumn{4}{|c|}{ Cyclotide } \\
\hline GRMZM2G032198 & Ch3 & P56879 & Chassalia parviflora \\
\hline GRMZM2G374405 & Ch3 & P56879 & Chassalia parviflora \\
\hline GRMZM2G450866 & Ch7 & P85233 & Viola hederacea \\
\hline
\end{tabular}

\subsection{Phylogenetic Analysis and Protein Motif Detection of Maize AMPs}

Multiple sequence alignments of the 46 maize AMPs were generated by using the software Muscle [26,27] and displayed by MSA software (http:/ / msa.biojs.net/) (Figure S1). The phylogenetic 
tree of the maize AMP families was constructed with Muscle and displayed with MEGA7 [28]. The maize AMPs appeared to be classified into 10 groups (Figure 1). Most of the maize AMPs fell into the predicted six groups including hevein, snakin, LTP, defensin, beta-barrelin, and cyclotide. Some of the maize AMPs, such as GRMZM2G062527P3, GRMZM2G062527P4, GRMZM2G117942, and GRMZM2G117971, further separated into more groups. To validate whether the identified maize AMPs carried the characteristic cysteine-rich motifs, each protein sequence was evaluated by using the bioinformatics web-server Pfam [29]. Each of these maize genes were verified to contain sufficient sequence similarity to cysteine-rich motifs found within known AMP models according to the Pfam web-server. These maize AMPs were then grouped into appropriate AMP gene families based on Pfam results (data not shown). The web-server software WebLogo [30,31] was used to further illustrate the characteristic cysteine-rich motif of these maize AMP sequences (Figure 2). Highly conserved amino acids within the WebLogo arrangement are depicted as relatively larger single letter code characters. From the WebLogo software, this arrangement reveals the highly conserved cysteine residues within the maize sequences across all groupings. The nature of this highly conserved cysteine-rich motif implies they have the capability of forming the characteristic multiple disulfide bonds found within AMPs (Figure 2, Supplemental Material). In addition to these findings, a conserved leucine-rich motif was observed. This motif preceded the cysteine-rich motif in the WebLogo graph (Figure 2).

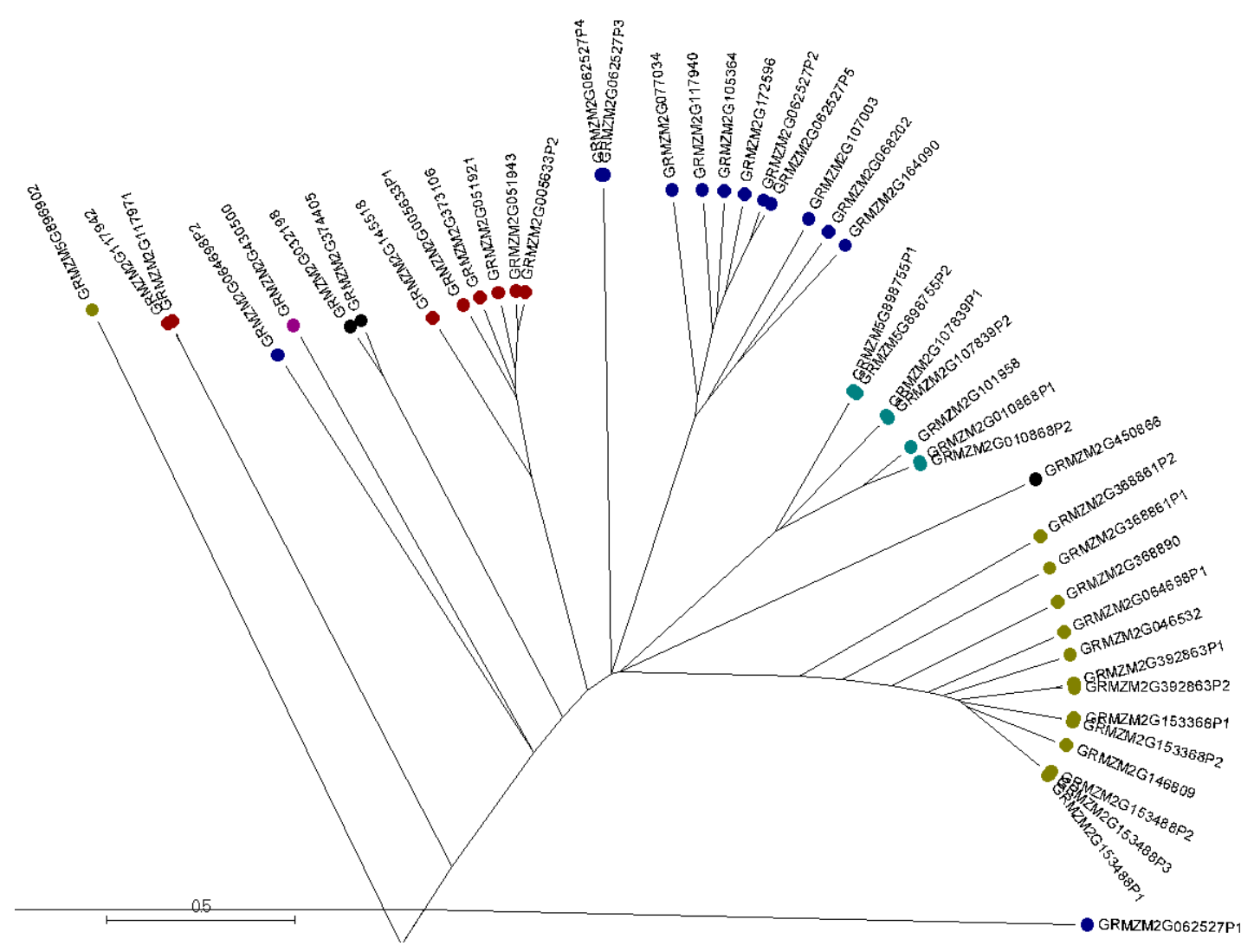

Figure 1. A phylogenetic tree of maize antimicrobial peptides. The forty-six identified maize AMPs are separated into 10 groups. Color coding indicates the corresponding AMP families with Hevein (red), Snakin (blue), Defensin (yellow-green), cyclotide (black), beta-barrelin (purple), and lipid transfer protein (LTP) (aqua). The phylogenetic tree is displayed with software MEGA 7. 

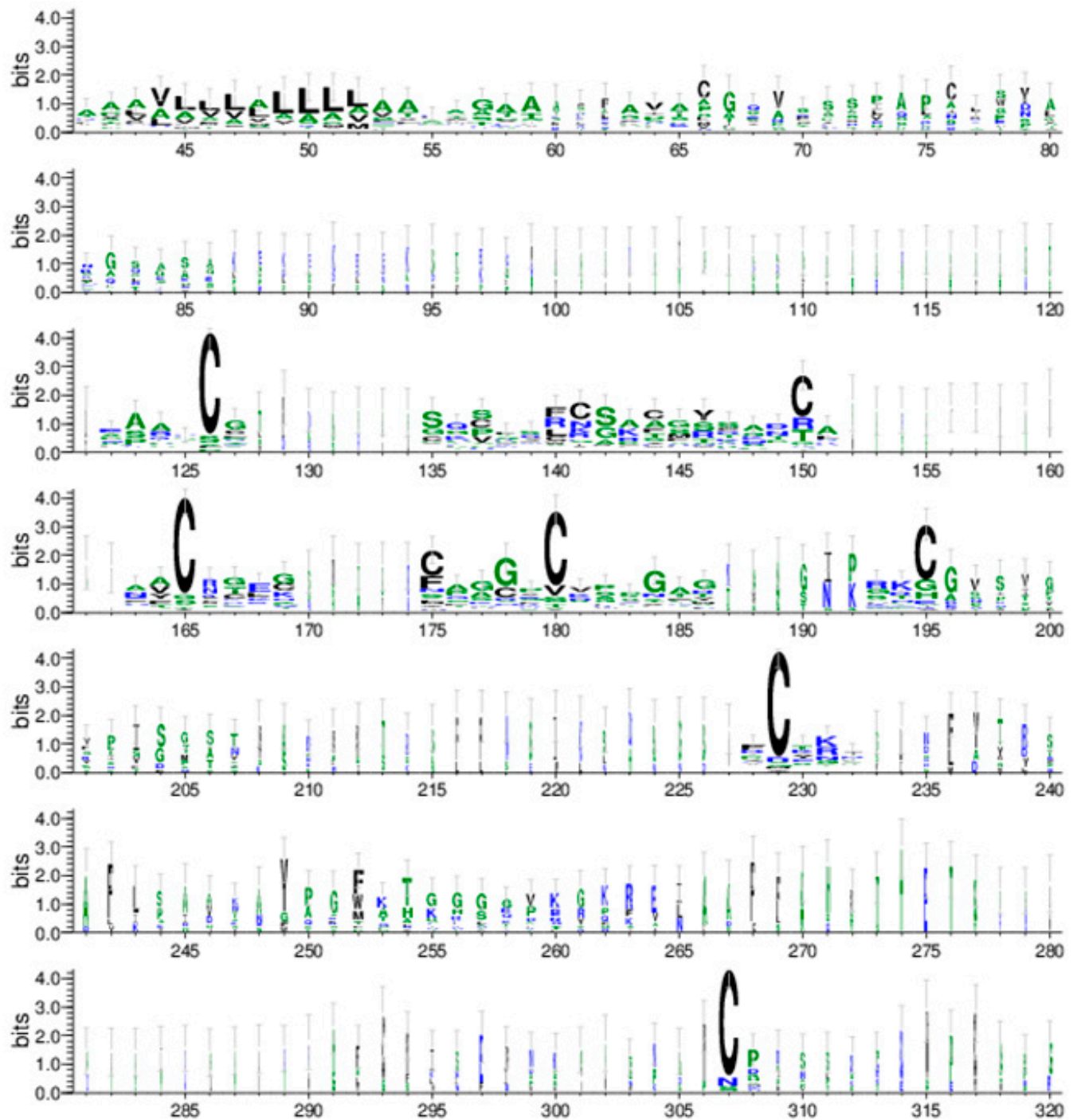

Figure 2. A WebLogo graph showing the positions of highly conserved cysteine-rich motifs observed among all forty-six maize AMP sequences. The degree of conservation is indicated by the relative size of the single-letter amino acid code.

\subsection{Expression Analysis of Maize AMP Genes and Polymorphism Study of Their Genomic Sequences}

To determine the functions of maize AMPs, the gene expression levels of maize AMPs were investigated by qRT-PCR using 10 maize inbred lines carrying different levels of fungus or insect resistance. Five of the maize inbred lines (Mp313e, Mp420, Mp715, Mp717, and Mp719) are resistant to the fungus Aspergillus flavus but susceptible to insect pests such as southwestern corn borer and fall armyworm. The other five maize inbred lines (Mp707, Mp708, Mp713, Mp714, and Mp716) carry insect resistance but fungus susceptibility. These maize inbred lines are not all derived from the same pedigrees. Various molecular defense pathways may exist while common resistance mechanism may also be possible. To identify which maize AMPs were involved with the observed resistance, maize leaf samples were collected from field corn plants in a randomized experimental design with three replications for each maize inbred line. Figure 3 displays a boxplot showing the summary of gene expression data from a group of 14 AMP genes that represented maize hevein, defensin, snakin, LTP, and cyclotide groups. The qRT-PCR data revealed variations in gene expression levels of the maize AMPs. The qRT-PCR data was subjected with statistical analysis by SAS program. Five maize AMPs were found significantly different in gene expression levels between distinct maize fungus and insect 
resistance groups. Figure 4 shows bar graphs for four of the significant differentially expressed maize AMP genes. The observed expression of GRMZM2G146809 (a defensin on maize chromosome 10) reveals a dichotomy between the two sets of maize inbred lines color-grouped according to their distinct types of resistance. Within the set of maize inbred lines associated with fungal resistance and insect susceptibility, the overall expression of GRMZM2G146809 was observed to be significantly greater than the insect resistant and fungal susceptible set of maize inbred lines $(p=0.01)$. Relative Expression of Lipid Transfer Protein GRMZM5G898755 (located on maize chromosome 10) displayed the widest range across the 10 maize inbred lines (Figure 3). The highest expression was observed in Mp420, fungal resistant, and the lowest expression was observed in Mp713, insect resistant (Figure 4). Additionally, the mean $\Delta \mathrm{Cp}$ of each maize inbred line within the set associated with fungal resistance and insect susceptibility was greater than those associated with insect resistance and fungus susceptibility. The relative expression of GRMZM2G368861 (located on maize chromosome 10) showed similar patterns associated with fungal resistance. Although the expression of GRMZM2G368861 between the two sets of maize inbred lines with contrary resistance type was significantly different, there was less variation in the overall expression of GRMZM2G368861 within the groups. Expression of maize Hevein GRMZM2G117971 (on maize chromosome 4) varied greatly across the 10 maize inbred lines. The highest mean value was associated with insect resistance. Although insect resistant Mp714 displayed the lowest relative expression, different insect resistance mechanisms may exist among the maize inbred lines. This result makes hevein GRMZM2G117971 the only significant maize AMP reported here to be expressed significantly greater in the group of maize inbred lines associated insect resistance and fungal susceptibility. Gene specific DNA markers were designed for the differentially expressed maize AMPs from maize genomic DNA in order to promote marker-assisted breeding application. DNA polymorphisms so far were observed among the 10 maize inbred lines including defensin GRMZM2G146809 and cyclotide GRMZM2G032198 (Figure 5).

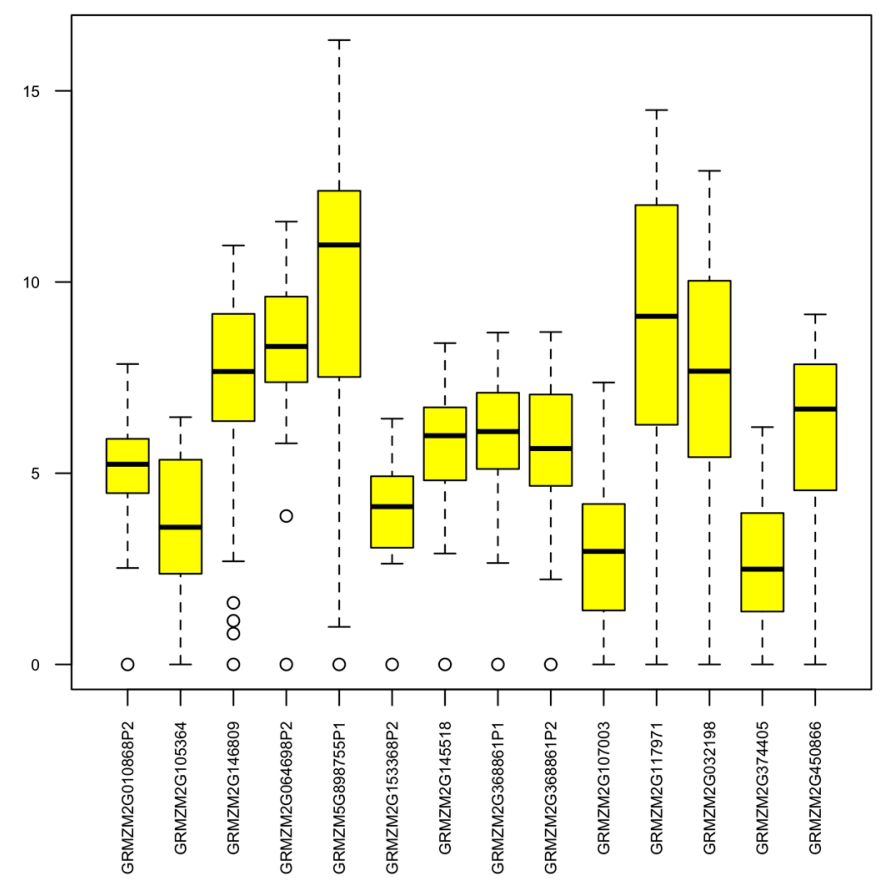

Figure 3. A boxplot showing the statistical summary of the range of relative gene expression levels of selected maize AMP genes across all 10 maize inbred lines. 

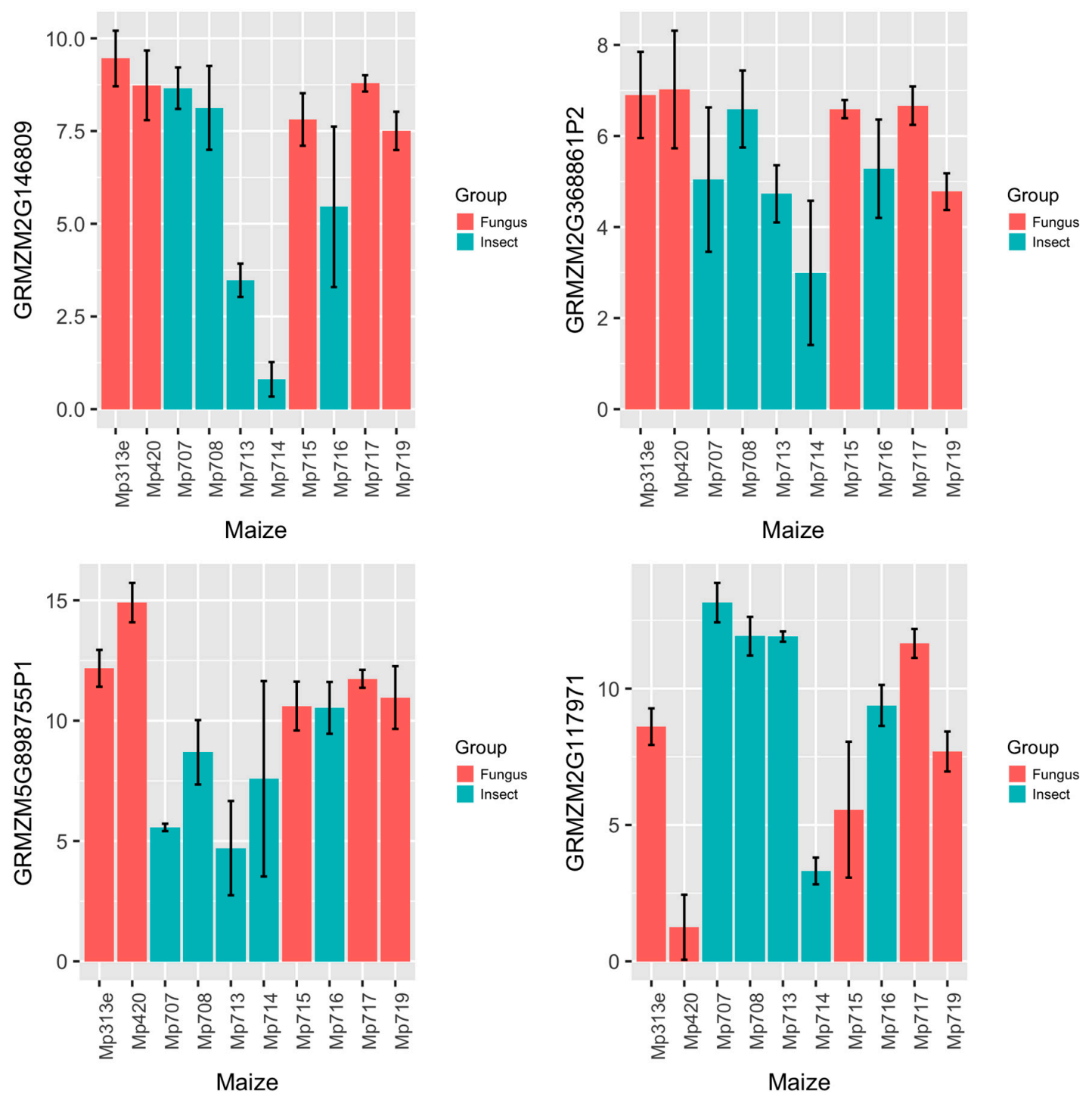

Figure 4. Bar graphs showing the relative gene expression levels in maize inbred lines that grouped by fungus resistance or insect resistance. The qRT-PCR data revealed variations in gene expression levels of the maize AMPs with distinct maize fungus and insect resistance groups. The expression of GRMZM2G146809 (a defensin on maize chromosome 10) reveals a dichotomy between the two sets of maize inbred lines. Relative Expression of Lipid Transfer Protein GRMZM5G898755 (located on maize chromosome 10) displayed significant variations across the 10 maize inbred lines. The relative expression of GRMZM2G368861 (located on maize chromosome 10) showed similar patterns associated with fungal resistance. Expression of maize Hevein GRMZM2G117971 (on maize chromosome 4) varied greatly across the 10 maize inbred lines and averaged higher in expression associated with insect resistance. 


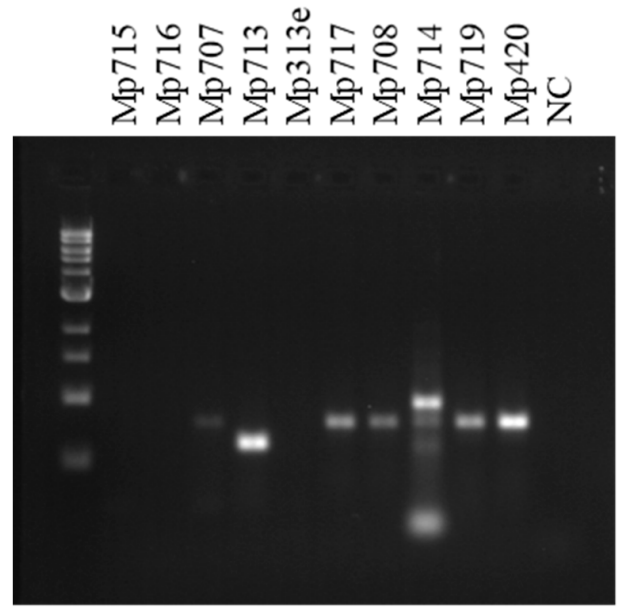

GRMZM2G146809

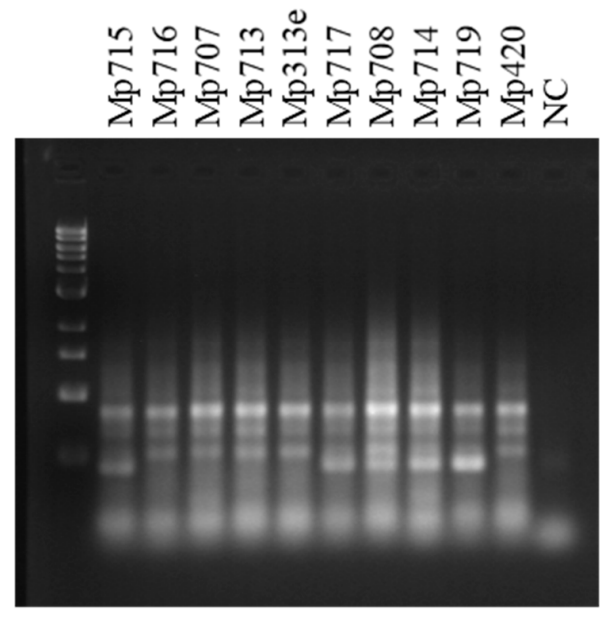

GRMZM2G032198

Figure 5. An agarose gel image showing DNA polymorphisms for two maize AMP genes. Gene specific DNA markers were designed for the differentially expressed maize AMPs from maize genomic DNA in order to promote marker-assisted breeding application. DNA polymorphisms were observed among the 10 maize inbred lines in defensin GRMZM2G146809 and cyclotide GRMZM2G032198.

\section{Discussion}

The most prominent characteristic of AMPs is their structural topology. This circular, or near circular, topology allows AMPs to withstand extreme proteolytic environments $[1,4,12,20]$. For example, the topology of cyclotides confers such a high degree of stability that a harsh microwave-based extraction methodology can be found in literature [32]. Although many families of AMPs can be differentiated by their structural topologies, plant AMPs are largely classified by their highly conserved cysteine-rich motifs $[1-3,11]$. Similar observations were confirmed from the identified maize AMPs by using multiple sequence alignment analysis subjected to the HMM pattern characterization algorithms (Figure 2 and Supplemental Material) [33]. Using Pfam, each maize sequence was characterized with cysteine-rich motifs. The WebLogo software further elucidated these motifs. From this arrangement, the highly conserved cysteine residues were distinguishable thereby implicating which residues can form multiple disulfide bonds (Figure 2, Supplemental Material). The antimicrobial activity of AMPs is dependent upon these disulfide bonds [2].

Despite the well-documented roles of plant AMPs in antimicrobial resistance, the biological functions of many plant AMPs remain largely unknown [1-3,11,34]. The fundamental principle underlying popular theories regarding AMPs and their mechanism of action revolves around the penetration and aggregation of AMPs into the microbial membrane of pathogens [1-3,12]. This penetration is facilitated by their amphipathic nature, a commonality among cysteine-rich proteins $[1-3,35]$. This feature is especially true for cyclotides as they are correlated with hydrophobicity $[1-4,8,9]$. The interaction of AMPs with these microbial membranes results in the formation of pore-like structures that are conducive to cell lysis through unregulated influx/efflux of essential ions.

Interestingly, a hydrophobic patch preceding the cysteine-rich motif was observed using the WebLogo software. Although literature reports hydrophobic patches are involved with the penetration of the microbial cell membrane $[1,9,10,14,22,32]$, a leucine-rich motif was observed in the maize sequences. In addition to these conserved leucine residues, other hydrophobic residues were observed in this hydrophobic patch, namely alanine and to a lesser extent valine. Prior to this finding, no literature reports highly conserved leucine residues within the hydrophobic patches of AMPs. As a result, a leucine-rich motif may be characteristic of hydrophobic patches in maize AMPs. 
Although many AMPs are a part of the constitutive defense $[1-3,7,10,36]$, insecticidal proteins were of significant interest in this study due to the relatively limited literature available. To investigate these oftentimes inducible AMPs, only plant tissue that was directly wounded by insect feeding was collected. Some AMPs, such as snakin-2, are both constitutive and locally expressed in response to wounding $[1,34]$. Others have also been reported to be expressed relative to the release of hormones, such as thionin in response to methyl jasmonate $[1,6,7,37]$. Therefore, the wounding of plant tissue prior to collection was considered essential for the relative expression analysis of AMPs within the panel of maize inbred lines analyzed in this study.

Out of the 189 plant AMPs reviewed from the PhytAMP database, 13 plant AMP sequences representing six AMP gene families that originated from nine plant species were used to identify sequences with high similarities in Maize B73 genome. Thirty-nine new maize AMPs were identified in this study in addition to 7 known maize AMPs documented in the PhytAMP database. All 46 maize AMPs fall into 10 groups by phylogenetic analysis, with six major groups in accordance to the AMP families of hevein, snakin, LTP, defensin, beta-barrelin, and cyclotide (Figure 1). Four other maize AMP groups each constitutes one or two maize AMPs were also identified at positions apart from the major maize AMP groups which separated earlier on the phylogenetic tree (Figure 1). Interestingly, maize AMPs were found to distribute on each of the 10 maize chromosomes with some loci located close to maize transposable element active regions. Given the vast antimicrobial functions of plant AMPs $[1,2,5,11]$, the genome-wide investigation of maize AMPs is of great importance to reveal naturally occurring maize resistance genes for elimination of plant diseases and improvement of maize production.

Out of the five original Plant AMPs that identified five significant maize AMPs associated with either fungus resistance or insect resistance, Q8H950 from Eutrema wasabi (maize counterpart GRMZM2G117971) was listed with antibacterial and antifungal functions in the PhytAMP Database, P56879 from Chassalia parviflora (maize counterpart GRMZM2G032198) was listed with antibacterial, antifungal, and insecticidal functions (Table 1, Figure 4). P81008 from Zea mays is a sodium channel blocker. Four maize AMPs similarly matched this sequence in the maize genome. On the other hand, Q2XX14 from Zea mays (with four new maize counterparts, including GRMZM2G898755P1) and Q9ZUL7 from Arabidopsis thaliana (maize counterpart GRMZM2G146809) were previously listed with unknown functions. In this study, we performed a gene expression analysis of maize AMP genes. Using a panel of 10 maize inbred lines carrying contrast levels of fungal or insect resistance, this study provided a gateway method to identify the potential functions of maize AMPs in plant defense systems.

Maize genome database data-mining allowed the identification of 46 maize AMP gene sequences. The number of maize AMP sequences varied from one in the beta-barrelin family to 13 in the defensin family. This is largely similar to the amount and types of AMPs distributed in other plant species. The maize AMP genes were found to distribute across the maize genome. One to three different maize AMP families were found on each maize chromosome (Table 1). The phylogenetic tree revealed, in many cases, that two or three maize AMP genes clustered together due to the presence of shorter versions of protein sequences derived from the same maize AMP gene (Figure 1). This indicated that alternative mRNA slicing mechanisms may exist in these maize AMP genes. The functional implications of such arrangements of the AMP genes in maize genome will be the subjects of future studies. In summary, our study identified 46 maize AMP sequences, four of them showing significance associated with fungal resistance and one showing significance related to insect resistance. This study also revealed polymorphisms in maize AMP genomic sequences that will be valuable for maize breeding applications. Together, these new findings will facilitate the identification of new natural plant resistance sources to confer fungus and insect resistance in maize. Future work based on the identification of maize AMP genes will focus on the extraction and characterization of maize AMPs to gain insights on the mechanisms and the antipathogenic peptide profiles in maize germplasm related to Aspergillus flavus and Spodoptera frugiperda resistance. 


\section{Materials and Methods}

\subsection{Plant Material}

Five maize (Zea mays) inbred lines with insect resistance and fungus susceptibility (Mp707, Mp708, Mp713, Mp714, and Mp716) as well as five maize inbred lines with insect susceptibility and fungus resistance (Mp313e, Mp420, Mp715, Mp717, and Mp719) were grown in field plots at the R.R. Foil Plant Science Research Center at Mississippi State University. The insect resistant inbred lines were selected based on the resistance to leaf-feeding by fall armyworm (Spodoptera frugiperda). Plants were infested with 30 larvae per maize plant during the mid-whorl stage of growth. Fourteen days after infestation, sections of leaves damaged by insect feeding were cut from maize plants and frozen in liquid nitrogen. Maize leaf samples were ground into powder via motor and pestle with the aid of liquid nitrogen. Samples were then stored at $-20^{\circ} \mathrm{C}$.

\subsection{DNA Extraction}

Extraction of plant DNA samples was performed using a DNeasy ${ }^{\circledR}$ Plant Mini Kit from Qiagen ${ }^{\circledR}$ with minor modifications from the manufacturer manual. Approximately $200 \mathrm{mg}$ of ground leaf tissue was transferred to pre-chilled centrifuge tubes using pre-chilled spatulas. In each sample tube, $400 \mu \mathrm{L}$ of buffer AP1 and $4 \mu \mathrm{L}$ RNase A were added. These were vortexed twice and incubated in a water bath at $65^{\circ} \mathrm{C}$ for $5 \mathrm{~min}$. The lysate was centrifuged for $1 \mathrm{~min}$ at 12,000 rpm and the supernatant was transferred to a fresh tube. The rest of the extraction was performed per the manufacturer protocol. Samples were then quantified using a Nanodrop2000c spectrophotometer (Thermo Fisher Scientific, Wilmington, DE, USA). The purity of the products was assessed via their $\mathrm{A}_{260} / \mathrm{A}_{280}$ values. DNA samples were stored at $-20^{\circ} \mathrm{C}$.

\subsection{Database Search for Maize (Zea mays) Antimicrobial Peptide Gene and Protein Sequences}

Maize antimicrobial peptide sequences were obtained using various databases. First, plant AMP sequences from various species in the PhytAMP Database (phytamp.pfba-lab-tun.org/) were used to search the UniProt protein database (uniport.org/). Amino acid and nucleic acid sequences were collected from the seven superfamilies-Cyclotide, Defensin, Hevein, Knottin, Lipid-transfer protein, Snakin, and Thionins based on the classifications in the PhytAMP Database. The basic alignment search tool (BLAST) was used to search for similar antimicrobial peptide sequences in MaizeGDB B73 genome database (maizegdb.org/). The corresponding nucleic acid sequence was acquired in addition to the amino acid sequence and their physical locations on maize chromosomes were also determined from MaizeGDB B73 genome database. Each protein sequence was then subjected to Pfam for motif identification (pfam.xam.org/). Regions containing AMP domains were later used for primer design.

\subsection{Design of Genomic DNA and cDNA Primers for Maize AMPs}

Nucleic acid sequences gathered from MaizeGDB gene regions containing AMP domains were used as templates for primer design. The Primer3 online software (http:/ / bioinfo.ut.ee/primer3-0.4.0/ primer3/) was used to design primers. Primers were designed such that product size ranged between 400 and $800 \mathrm{bp}$ for genomic DNA. The cDNA sequence representing the mature mRNA sequence for the gene of interest was used as the template for the design of primers used in quantitative real-time polymerase chain reaction (qRT-PCR). cDNA primers were designed using the primer3 online software such that products were approximately $150 \mathrm{bp}$. DNA oligos were synthesized by Sigma Life Sciences (Sigma-Aldrich, Inc., St. Louis, MO, USA) (Table 2). 
Table 2. Polymerase chain reaction (PCR) primers for cDNA and genomic DNA of maize AMP genes.

\begin{tabular}{|c|c|}
\hline Maize Genes & Primers \\
\hline \multicolumn{2}{|l|}{ cDNA Primers } \\
\hline \multicolumn{2}{|r|}{ Hevein } \\
\hline GRMZM2G117942F & TACATCGATCGGTTGCCAAA \\
\hline GRMZM2G117942R & TTCTGCTGCGGGTTGTAGA \\
\hline GRMZM2G145518F & TTCTCCAAGCACAGGAGACA \\
\hline GRMZM2G145518R & ACGCCTCACTTCCCACTGTA \\
\hline GRMZM2G117971F & TATGGATGTGATCCCACACG \\
\hline GRMZM2G117971R & AGTGGACGACACATATTCGAGA \\
\hline \multicolumn{2}{|r|}{ Snakin } \\
\hline GRMZM2G105364F & TGGAATGCTACCAGCCAGAT \\
\hline GRMZM2G105364R & CGGGATGTTCCTCATCAATC \\
\hline GRMZM2G172596F & CTGCTCCTCTGCTTCCTGTT \\
\hline GRMZM2G172596R & GTTCTTGTAGCCCTCGTGCTT \\
\hline GRMZM2G107003F & CGCCACGTTTTGTATGATCC \\
\hline GRMZM2G107003R & ACACAGACCCATCAACGTCA \\
\hline \multicolumn{2}{|r|}{ LTP } \\
\hline GRMZM2G101958F & CATATGTGACCGTGTGTTCCA \\
\hline GRMZM2G101958R & CTCGCCCAGCTTTGTTTTAT \\
\hline GRMZM2G010868P1F & TTGGCACCAAGCACTAAAGA \\
\hline GRMZM2G010868P1R & TCССАAАТСАТССССТАGAA \\
\hline GRMZM2G010868P2F & ССТGCAACTGCCTCAAGAAC \\
\hline GRMZM2G010868P2R & TGCATGCATACTACССТАССТG \\
\hline GRMZM5G898755P1F & TCGACTGCACCAAGATCAAC \\
\hline GRMZM5G898755P1R & TCTGATGCATGACACACACG \\
\hline GRMZM5G898755P2F & AGCAGCACCTCAATGTCCTT \\
\hline GRMZM5G898755P2R & CATGCATATGTACGGCGAAT \\
\hline GRMZM2G107839P1F & CTCCGGTTTGCAGAAACAAC \\
\hline GRMZM2G107839P1R & CTAGGCATCAGCACAGTCCA \\
\hline GRMZM2G107839P2F & GATCCACCTACTTGTTCAGACAG \\
\hline GRMZM2G107839P2R & CATCTCCTCTGATCGTCCTTT \\
\hline \multicolumn{2}{|r|}{ Defensin } \\
\hline GRMZM2G368890F & GCCGGAATATGTGGACGAT \\
\hline GRMZM2G368890R & ACATGCAGACCCCCTTGAA \\
\hline GRMZM2G392863P1F & TGTTGTACGTACGTCTGCCTCT \\
\hline GRMZM2G392863P1R & AACAATCAGCGTCGTCTCTT \\
\hline GRMZM2G392863P2F & CCGCTGAGATCCTAGGAAGA \\
\hline GRMZM2G392863P2R & CTGATGAGTCCACAGCACAGA \\
\hline GRMZM2G146809F & GGTCCGTTTGCGTTTGTTTC \\
\hline GRMZM2G146809R & GGTTCATCAATGCAACGAGAC \\
\hline GRMZM5G896902F & AGAAGGACAGCGAGCGATT \\
\hline GRMZM5G896902R & CCGGGAGTAGGTTAATTTAGCA \\
\hline GRMZM2G153488P1F & GTTGTACTTTCTGCATCCGTTG \\
\hline GRMZM2G153488P1R & TTGGTCATCAAGTTCCCTAGC \\
\hline GRMZM2G153488P2F & AGCCTTACGTAGCGAAGCTC \\
\hline GRMZM2G153488P2R & AGCAACGAGGAGTTGAGTCG \\
\hline GRMZM2G153488P3F & ATAAACCGTGGCTCTGGTTC \\
\hline GRMZM2G153488P3R & TTGCTCTGAGCTTCGCTACG \\
\hline GRMZM2G064698P1F & AGTTCGTGAATCCCTGAAGC \\
\hline GRMZM2G064698P1R & ATTCCCTTGCCTGTGCCATA \\
\hline GRMZM2G368861P1F & GATAGTGACGTACGCGCAAC \\
\hline GRMZM2G368861P1R & GCATACGATCTGACGCTCAT \\
\hline GRMZM2G368861P2F & GCGATGGAGCTCATCAAGTC \\
\hline GRMZM2G368861P2R & GTCCATGAGGCAGCAGAAAT \\
\hline GRMZM2G046532F & GGTGCCCATACCATAGCTTC \\
\hline GRMZM2G046532R & TAACAAACGAGCAGGAGGAG \\
\hline GRMZM2G064698P2F & AGTTCGTGAATCCCTGAAGC \\
\hline GRMZM2G064698P2R & ATTCCCTTGCCTGTGCCATA \\
\hline GRMZM2G153368P1F & AAGAAGCCTTGCTAGTTCATCG \\
\hline GRMZM2G153368P1R & CCCAGCAATTTAAGGACTGC \\
\hline GRMZM2G153368P2F & GTACGTACTCGTACCAGGCAGA \\
\hline GRMZM2G153368P2R & GCATGGCTACTCCCATTTTG \\
\hline
\end{tabular}


Table 2. Cont.

\begin{tabular}{cc}
\hline \multicolumn{1}{c}{ Maize Genes } & Primers \\
\hline & $\beta$-Barrelin \\
\hline GRMZM2G430500F & CTCGGGGGATACGTCGAT \\
GRMZM2G430500R & TGGGTGTCCTCGAAAACTTG \\
\hline & GTGTTTGGCCTGGACTTCAT \\
\hline GRMZM2G032198F & GGCGTCACGAGTTTATTTCA \\
GRMZM2G032198R & GTCCCCTGTTTGAATCCTG \\
GRMZM2G374405F & TTCACACGTAACGGGATCAG \\
GRMZM2G374405R & GGGCTTGTTGCAGTGGTAGT \\
GRMZM2G450866F & CGATCTTGTGACGGTTCAGC \\
GRMZM2G450866R & \\
\hline Genomic DNA Primers & Defensin \\
\hline GRMZM2G146809F2 & GGCCAAGTATACTCGCCAGA \\
GRMZM2G146809R2 & TCGAAGGGTTATTGCATTCC \\
\hline & Cyclotide \\
\hline GRMZM2G032198F2 & GTTGGGAGCAAAGCAAAGAG \\
GRMZM2G032198R2 & GAGGAGCAGGCGATTGAGTA \\
\hline
\end{tabular}

\subsection{Polymerase Chain Reaction for Genomic DNA}

One microliter of DNA sample was added into $24 \mu \mathrm{L}$ of PCR reaction mixture containing $1 \times$ ThermoPol Reaction Buffer from New England BioLabs (New England BioLabs, Inc., Ipswich, MA, USA), $200 \mu \mathrm{M}$ dNTP from New England BioLabs, $1 \mu \mathrm{M}$ forward primer, $1 \mu \mathrm{M}$ reverse primer, and 2.5 units Taq polymerase $/ 50 \mu \mathrm{L}$ PCR. For each set of reaction mixtures, $1 \mu \mathrm{L}$ of $\mathrm{ddH}_{2} \mathrm{O}$ was used as a control. Upon starting, the thermocycler held an initial denaturation for $3 \mathrm{~min}$ at $95^{\circ} \mathrm{C}$. Afterwards, the PCR was performed with a denaturation step for $45 \mathrm{~s}$ at $94{ }^{\circ} \mathrm{C}$, an annealing step of $50 \mathrm{~s}$ at $52{ }^{\circ} \mathrm{C}$, and an elongation step at $72{ }^{\circ} \mathrm{C}$ for $80 \mathrm{~s}$ for 40 cycles. A final extension lasted for $10 \mathrm{~min}$ at $72{ }^{\circ} \mathrm{C}$ before a final hold step at $4{ }^{\circ} \mathrm{C}$. Amplification of PCR products was determined via agarose gel electrophoresis using $1 \%(w / v)$ agarose in $1 \times$ TAE buffer. $1 \mathrm{~kb}$ DNA ladder from New England BioLabs (New England BioLabs, Inc., Ipswich, MA, USA) was used. The electrophoresis was conducted at $75 \mathrm{~V}$ on constant voltage for $75 \mathrm{~min}$. Agarose gels were then stained with ethidium bromide for $60 \mathrm{~min}$ and de-stained in water for 10-60 min prior to viewing with a UV gel-imager.

\subsection{RNA Extraction}

RNA was extracted using the spin column protocol with minor modifications according to the Aurum $^{\mathrm{TM}}$ Total RNA Fatty and Fibrous Tissue Kit from Bio-Rad (Bio-Rad Laboratories, Inc., Hercules, CA, USA). To start the extraction, frozen leaf tissue was ground to a fine powder using mortar and pestle with liquid nitrogen. In $2.0 \mathrm{~mL}$ microfuge tubes, $100 \mathrm{mg}$ of tissue was suspended in $1 \mathrm{~mL}$ PureZOL, vortexed for $1 \mathrm{~min}$, and incubated at room temperature for $5 \mathrm{~min}$. Samples were then pelleted at $12,000 \times \mathrm{g}$ for $5 \mathrm{~min}$ at $4{ }^{\circ} \mathrm{C}$. After transferring the supernatant to new $2.0 \mathrm{~mL}$ tubes, $200 \mu \mathrm{L}$ of chloroform was added to the lysate. The sample was then centrifuged at $12,000 \times g$ for $15 \mathrm{~min}$ at $4{ }^{\circ} \mathrm{C}$. The aqueous phase was then transferred to new $2.0 \mathrm{~mL}$ tubes without disturbing the interphase. Equal volume $70 \%$ ethanol was then added to the sample and mixed via pipetting. Then the manufacturer protocol was followed to finish the extraction. A volume of $30 \times$ of the elution buffer was used to elute each RNA sample via centrifugation for $2 \mathrm{~min}$ at $12,000 \times g$.

\subsection{Synthesis of $c D N A$ and $q R T-P C R$ Analysis}

The synthesis of cDNA was completed using the ThermoScript ${ }^{\mathrm{TM}}$ RT-PCR System kit from Life Technologies (Life Technologies Corporation, Carlsbad, CA, USA) and the manufacturer protocol was followed. The RNA was denatured at $65^{\circ} \mathrm{C}$ for $5 \mathrm{~min}$ and then held at $4{ }^{\circ} \mathrm{C}$. The synthesis of cDNA 
was carried out at $50{ }^{\circ} \mathrm{C}$ for $60 \mathrm{~min}, 85^{\circ} \mathrm{C}$ for $5 \mathrm{~min}$, and then held indefinitely at $4{ }^{\circ} \mathrm{C}$. The cDNA samples were then stored in $-20^{\circ} \mathrm{C}$. For qRT-PCR, the LightCycler ${ }^{\circledR} 480$ SYBR Green I Master enzyme mix (Roche Diagnostics Corporation, Indianapolis, IN, USA) was used. The run protocol contained one denaturation step at $95^{\circ} \mathrm{C}$ for $5 \mathrm{~min}$, followed by 45 cycles of $95^{\circ} \mathrm{C}$ for $10 \mathrm{~s}, 60^{\circ} \mathrm{C}$ for $15 \mathrm{~s}$, and $72{ }^{\circ} \mathrm{C}$ for $15 \mathrm{~s}$. The final step was a $10 \mathrm{~s}$ cool down to $40{ }^{\circ} \mathrm{C}$. To determine the efficiency of the amplification, primer efficiency was evaluated by diluting cDNA using a five-step 3-fold serial dilution. The coefficient of determination $\left(R^{2}\right)$ from the linear regression of $\Delta \mathrm{Cp}$ and $\log (1 /$ dilution factor) was retrieved. Following this, the efficiency was calculated by $\log _{2}(r+1)$, where $r=R^{2}$. Primers with the efficiency above 0.7 were used for qRT-PCR analysis of gene expression levels on the ten maize inbred lines. The expression level of GAPDH gene from each sample was evaluated and used as a house-keeping control gene for normalization purpose.

\subsection{Statistical and Bioinformatics Analysis}

For qRT-PCR analysis of gene expression, the $\mathrm{Cp}$ values were normalized and used for statistical analysis. Amplification efficiencies of cDNA primers were incorporated to calculate the $\mathrm{Cp}$ values. The expression level of glyceraldehyde 3-phosphate dehydrogenase (GAPDH) was used as a house-keeping gene control for the data normalization. SAS University Edition for Windows (SAS Institute Inc., Cary, NC, USA) was used to conduct ANOVA analysis for gene expression data. Least Significant Difference tests (LSDs) at $p<0.05$ and $p<0.01$ levels were performed for estimate of the significance of differential gene expression. Statistics results were graphed with the R package ggplot2 (http:/ / ggplot2.org/). Multiple sequence alignments were generated by using the software Muscle [23,24] and displayed by MSA (http:/ / msa.biojs.net/). The phylogenetic tree was constructed with Muscle and displayed with Mega 7 [25]. To validate the characteristics of the maize AMP motifs, the bioinformatics web-server Pfam was used [26]. The web-server software WebLogo [27,28] was used to illustrate the characteristic protein motifs.

Supplementary Materials: Supplementary materials can be found at www.mdpi.com/1422-0067/18/9/1938/s1.

Acknowledgments: This work was sponsored by Specific Cooperative Agreement (No. 58-6406-1-600) of USDA Agricultural Research Service (ARS) and Mississippi Agricultural and Forestry Experiment Station (MAFES) at Mississippi State University. It was also supported by the National Corn Growers Association and the Aflatoxin Mitigation Center of Excellence Aflatoxin Research Program.

Author Contributions: Xueyan Shan and William Paul Williams conceived and designed the experiments. Joseph Noonan, Xueyan Shan, and William Paul Williams performed the experiments. Joseph Noonan and Xueyan Shan analyzed the data. Joseph Noonan and Xueyan Shan wrote the manuscript. William Paul Williams contributed to and edited the manuscript. All authors have reviewed and approved the final manuscript.

Conflicts of Interest: The authors declare no conflict of interest.

\section{References}

1. Tam, J.P.; Wang, S.; Wong, K.H.; Tan, W.L. Antimicrobial Peptides from Plants. Pharmaceuticals 2015, 8, 711-757. [CrossRef] [PubMed]

2. Agriculture, R.G.; Canada, A. Host Defense Peptides and Their Potential as Therapeutic Agents; Epand, R.M., Ed.; Springer International Publishing: Cham, Switzerland, 2016; pp. 111-136.

3. Nawrot, R.; Barylski, J.; Nowicki, G.; Broniarczyk, J.; Buchwald, W.; Goździcka-Józefiak, A. Plant antimicrobial peptides. Folia Microbiol. 2014, 59, 181-196. [CrossRef] [PubMed]

4. Pinto, M.F.S.; Silva, O.N.; Viana, J.C.; Porto, W.F.; Migliolo, L.; da Cunha, N.B.; Gomes, N.; Fensterseifer, I.C.M.; Colgrave, M.L.; Craik, D.J.; et al. Characterization of a Bioactive Acyclotide from Palicourea rigida. J. Nat. Prod. 2016, 79, 2767-2773. [CrossRef] [PubMed]

5. Hammami, R.; Ben Hamida, J.; Vergoten, G.; Fliss, I. PhytAMP: A database dedicated to antimicrobial plant peptides. Nucleic Acids Res. 2009, 37, D963-D968. [CrossRef] [PubMed]

6. Goyal, R.K.; Mattoo, A.K. Multitasking antimicrobial peptides in plant development and host defense against biotic/abiotic stress. Plant Sci. 2014, 228, 135-149. [CrossRef] [PubMed] 
7. Herbel, V.; Sieber-Frank, J.; Wink, M. The antimicrobial peptide snakin-2 is upregulated in the defense response of tomatoes (Solanum lycopersicum) as part of the jasmonate-dependent signaling pathway. J. Plant Physiol. 2017, 208, 1-6. [CrossRef] [PubMed]

8. Ravipati, A.S.; Poth, A.G.; Troeira Henriques, S.; Bhandari, M.; Huang, Y.-H.; Nino, J.; Colgrave, M.L.; Craik, D.J. Understanding the Diversity and Distribution of Cyclotides from Plants of Varied Genetic Origin. J. Nat. Prod. 2017, 80, 1522-1530. [CrossRef] [PubMed]

9. Barbeta, B.L.; Marshall, A.T.; Gillon, A.D.; Craik, D.J.; Anderson, M.A. Plant cyclotides disrupt epithelial cells in the midgut of lepidopteran larvae. Proc. Natl. Acad. Sci. USA 2008, 105, 1221-1225. [CrossRef] [PubMed]

10. Craik, D.J. Host-defense activities of cyclotides. Toxins 2012, 4, 139-156. [CrossRef] [PubMed]

11. Gruber, C.W.; Cemazar, M.; Anderson, M.A.; Craik, D.J. Insecticidal plant cyclotides and related cystine knot toxins. Toxicon 2007, 49, 561-575. [CrossRef] [PubMed]

12. Troeira Henriques, S.; Craik, D.J. Cyclotide Structure and Function: The Role of Membrane Binding and Permeation. Biochemistry 2017, 56, 669-682. [CrossRef] [PubMed]

13. Weidmann, J.; Craik, D.J. Discovery, structure, function, and applications of cyclotides: Circular proteins from plants. J. Exp. Bot. 2016, 67, 4801-4812. [CrossRef] [PubMed]

14. Daly, N.L.; Gunasekera, S.; Clark, R.J.; Lin, F.; Wade, J.D.; Anderson, M.A.; Craik, D.J. The N-terminal pro-domain of the kalata B1 cyclotide precursor is intrinsically unstructured. Biopolymers 2016, 106, 825-833. [CrossRef] [PubMed]

15. Mulvenna, J.P.; Mylne, J.S.; Bharathi, R.; Burton, R.A.; Shirley, N.J.; Fincher, G.B.; Anderson, M.A.; Craik, D.J. Discovery of cyclotide-like protein sequences in graminaceous crop plants: Ancestral precursors of circular proteins? Plant Cell 2006, 18, 2134-2144. [CrossRef] [PubMed]

16. Nahirñak, V.; Rivarola, M.; Gonzalez de Urreta, M.; Paniego, N.; Hopp, H.E.; Almasia, N.I.; Vazquez-Rovere, C. Genome-wide Analysis of the Snakin/GASA Gene Family in Solanum tuberosum cv. Kennebec. Am. J. Potato Res. 2016, 93, 172-188. [CrossRef]

17. Kaur, J.; Fellers, J.; Adholeya, A.; Velivelli, S.L.S.; El-Mounadi, K.; Nersesian, N.; Clemente, T.; Shah, D. Expression of apoplast-targeted plant defensin MtDef4.2 confers resistance to leaf rust pathogen Puccinia triticina but does not affect mycorrhizal symbiosis in transgenic wheat. Transgenic Res. 2017, 26, 37-49. [CrossRef] [PubMed]

18. Menzel, L.P.; Chowdhury, H.M.; Masso-Silva, J.A.; Ruddick, W.; Falkovsky, K.; Vorona, R.; Malsbary, A.; Cherabuddi, K.; Ryan, L.K.; DiFranco, K.M.; et al. Potent in vitro and in vivo antifungal activity of a small molecule host defense peptide mimic through a membrane-active mechanism. Sci. Rep. 2017, 7, 4353. [CrossRef] [PubMed]

19. Vriens, K.; Cammue, B.; Thevissen, K. Antifungal Plant Defensins: Mechanisms of Action and Production. Molecules 2014, 19, 12280-12303. [CrossRef] [PubMed]

20. Wang, C.K.; Hu, S.-H.; Martin, J.L.; Sjögren, T.; Hajdu, J.; Bohlin, L.; Claeson, P.; Göransson, U.; Rosengren, K.J.; Tang, J.; et al. Combined X-ray and NMR analysis of the stability of the cyclotide cystine knot fold that underpins its insecticidal activity and potential use as a drug scaffold. J. Biol. Chem. 2009, 284, 10672-10683. [CrossRef] [PubMed]

21. Porto, W.F.; Miranda, V.J.; Pinto, M.F.S.; Dohms, S.M.; Franco, O.L. High-performance computational analysis and peptide screening from databases of cyclotides from poaceae. Biopolymers 2016, 106, 109-118. [CrossRef] [PubMed]

22. Craik, D.J.; Mylne, J.S.; Daly, N.L. Cyclotides: Macrocyclic peptides with applications in drug design and agriculture. Cell. Mol. Life Sci. 2010, 67, 9-16. [CrossRef] [PubMed]

23. Sousa, D.A.; Porto, W.F.; Silva, M.Z.; da Silva, T.R.; Franco, O.L. Influence of Cysteine and Tryptophan Substitution on DNA-Binding Activity on Maize $\alpha$-Hairpinin Antimicrobial Peptide. Molecules 2016, 21, 1062. [CrossRef] [PubMed]

24. Andorf, C.M.; Cannon, E.K.; Portwood, J.L.; Gardiner, J.M.; Harper, L.C.; Schaeffer, M.L.; Braun, B.L.; Campbell, D.A.; Vinnakota, A.G.; Sribalusu, V.V.; et al. MaizeGDB update: New tools, data and interface for the maize model organism database. Nucleic Acids Res. 2016, 44, D1195-D1201. [CrossRef] [PubMed]

25. Walley, J.W.; Sartor, R.C.; Shen, Z.; Schmitz, R.J.; Wu, K.J.; Urich, M.A.; Nery, J.R.; Smith, L.G.; Schnable, J.C.; Ecker, J.R.; et al. Integration of omic networks in a developmental atlas of maize. Science 2016, 353, 814-818. [CrossRef] [PubMed] 
26. Edgar, R.C. MUSCLE: Multiple sequence alignment with high accuracy and high throughput. Nucleic Acids Res. 2004, 32, 1792-1797. [CrossRef] [PubMed]

27. Edgar, R.C. MUSCLE: A multiple sequence alignment method with reduced time and space complexity. BMC Bioinforma. 2004, 5, 113. [CrossRef] [PubMed]

28. Kumar, S.; Stecher, G.; Tamura, K. MEGA7: Molecular Evolutionary Genetics Analysis Version 7.0 for Bigger Datasets. Mol. Biol. Evol. 2016, 33, 1870-1874. [CrossRef] [PubMed]

29. Finn, R.D.; Coggill, P.; Eberhardt, R.Y.; Eddy, S.R.; Mistry, J.; Mitchell, A.L.; Potter, S.C.; Punta, M.; Qureshi, M.; Sangrador-Vegas, A.; et al. The Pfam protein families database: Towards a more sustainable future. Nucleic Acids Res. 2016, 44, D279-D285. [CrossRef] [PubMed]

30. Crooks, G.E.; Hon, G.; Chandonia, J.M.; Brenner, S.E. WebLogo: A sequence logo generator. Genome Res. 2004, 14, 1188-1190. [CrossRef] [PubMed]

31. Schneider, T.D.; Stephens, R.M. Sequence logos: A new way to display consensus sequences. Nucleic Acids Res. 1990, 18, 6097-6100. [CrossRef] [PubMed]

32. Farhadpour, M.; Hashempour, H.; Talebpour, Z.; A-Bagheri, N.; Shushtarian, M.S.; Gruber, C.W.; Ghassempour, A. Microwave-assisted extraction of cyclotides from Viola ignobilis. Anal. Biochem. 2016, 497, 83-89. [CrossRef] [PubMed]

33. Eddy, S.R. Hidden Markov models. Curr. Opin. Struct. Biol. 1996, 6, 361-365. [CrossRef]

34. Berrocal-Lobo, M.; Segura, A.; Moreno, M.; López, G.; García-Olmedo, F.; Molina, A. Snakin-2, an antimicrobial peptide from potato whose gene is locally induced by wounding and responds to pathogen infection. Plant Physiol. 2002, 128, 951-961. [CrossRef] [PubMed]

35. Harris, P.W.R.; Yang, S.-H.; Molina, A.; López, G.; Middleditch, M.; Brimble, M.A. Plant antimicrobial peptides snakin-1 and snakin-2: Chemical synthesis and insights into the disulfide connectivity. Chemistry 2014, 20, 5102-5110. [CrossRef] [PubMed]

36. Games, P.D.; DaSilva, E.Q.G.; de Oliveira Barbosa, M.; Almeida-Souza, H.O.; Fontes, P.P.; DeMagalhães, M.J.; Pereira, P.R.G.; Prates, M.V.; Franco, G.R.; Faria-Campos, A.; et al. Computer aided identification of a Hevein-like antimicrobial peptide of bell pepper leaves for biotechnological use. BMC Genomics 2016, 17, 999. [CrossRef] [PubMed]

37. Epple, P.; Apel, K.; Bohlmann, H. An Arabidopsis thaliana thionin gene is inducible via a signal transduction pathway different from that for pathogenesis-related proteins. Plant Physiol. 1995, 109, 813-820. [CrossRef] [PubMed]

(C) 2017 by the authors. Licensee MDPI, Basel, Switzerland. This article is an open access article distributed under the terms and conditions of the Creative Commons Attribution (CC BY) license (http:/ / creativecommons.org/licenses/by/4.0/). 